\title{
IC9-GD2-CAR-CD28-OX40-expressing Autologous NKT Cells
}

\author{
National Cancer Institute
}

\section{Source}

National Cancer Institute. iC9-GD2-CAR-CD28-OX40-expressing Autologous NKT Cells. NCI Thesaurus. Code C148135.

A preparation of autologous interleukin-15 (IL-15)-expressing natural killer T-cells (NKT) transduced with a retroviral vector encoding a chimeric antigen receptor (CAR) derived from the antibody $14 \mathrm{G} 2 \mathrm{a}$ that recognizes disialog ang lioside GD2 (GD2-CAR) that is coupled to the co-stimulatory domains of CD28 and OX40 (CD134), and to the zeta chain of the TCR/CD3 complex (CD3-zeta), and linked to the suicide gene inducible caspase 9 (iCasp9 or iC9), with potential immunomodulating and antineoplastic activities. Upon transfusion, the iC9-GD2-CAR-CD28-OX40-expressing autologous NKT cells recognize, bind to and induce selective cytotoxicity in GD2-expressing tumor cells. The tumorassociated antigen (TAA) GD2 is overexpressed on the surface of neuroblastoma cells and by other neuroectoderm-derived neoplasms, while it is minimally expressed on normal, healthy cells. The iCasp9 safety switch consists of a full-length caspase 9, including its caspase recruitment domain, linked to a human FK506 drug-binding domain with an F36V mutation (FKBP12-F36V). If the administered NKT cells lead to unacceptable side effects, the chemical homodimerizer AP1903, which binds to the FKBP12-F36V drug-binding domain, activates caspase 9, and results in apoptosis of the administered NKT cells, can be administered. 